\title{
SimTalk: Simulation of IoT Applications
}

\author{
Yun-Wei Lin ${ }^{1}{ }^{\mathbb{D}}$, Yi-Bing Lin $^{2, *}$ and Tai-Hsiang Yen ${ }^{2}$ \\ 1 College of Artificial Intelligence, National Chiao Tung University (NCTU), Hsinchu 300, Taiwan; \\ jyneda@nctu.edu.tw \\ 2 Department of Computer Science, National Chiao Tung University (NCTU), Hsinchu 300, Taiwan; \\ ksoy.cs08g@nctu.edu.tw \\ * Correspondence: liny@nctu.edu.tw
}

Received: 7 April 2020; Accepted: 27 April 2020; Published: 30 April 2020

\begin{abstract}
The correct implementation and behavior of Internet of Things (IoT) applications are seldom investigated in the literature. This paper shows how the simulation mechanism can be integrated well into an IoT application development platform for correct implementation and behavior investigation. We use an IoT application development platform called IoTtalk as an example to describe how the simulation mechanism called SimTalk can be built into this IoT platform. We first elaborate on how to implement the simulator for an input IoT device (a sensor). Then we describe how an output IoT device (an actuator) can be simulated by an animated simulator. We use a smart farm application to show how the simulated sensors are used for correct implementation. We use applications including interactive art (skeleton art and water dance) and the pendulum physics experiment as examples to illustrate how IoT application behavior investigation can be achieved in SimTalk. As the main outcome of this paper, the SimTalk simulation codes can be directly reused for real IoT applications. Furthermore, SimTalk is integrated well with an IoT application verification tool in order to formally verify the IoT application configuration. Such features have not been found in any IoT simulators in the world.
\end{abstract}

Keywords: simulation; IoT; sensor; actuator; smart farm; interactive art; physics experiments

\section{Introduction}

Many smart applications have been developed with Internet of Things (IoT) technology, including home automation [1], a smart aquarium [2,3], an intelligent campus [4,5], precision agriculture [6,7], interactive art and entertainment [8-10], and more. However, it is seldom mentioned how they are correctly implemented, especially for the existing or envisioned applications in remote sensing. To develop these applications, simulations provide a cost-effective verification approach to end-to-end execution. Through simulation, we can also evaluate the conceivability of applying particular techniques to the target IoT applications, which shed light on directions for possible future implementation. We will elaborate on existing IoT simulation solutions in Section 2. These solutions provide guidelines for implementing the real IoT applications. However, the simulation codes of these solutions cannot be directly reused for real applications due to their "discrete event" nature. Furthermore, since the codes of the real applications are separately developed, it does not guarantee that the codes for real IoT applications are consistent with the simulation codes. An extra verification process is required.

To resolve this issue, this paper proposes SimTalk based on an IoT development platform called IoTtalk [11]. The underlying concept of SimTalk is to develop a time-driven simulation that can automatically translate the simulation codes to those for the real IoT applications and vice versa. Therefore, when we complete the simulation, the codes can be automatically translated into real IoT applications running on IoTtalk. 
IoTtalk offers a graphical user interface (GUI) to describe the relationship between sensors and actuators graphically, allowing simple data manipulation and transfer between IoT devices to occur. After an IoT service has been developed, IoTtalk GUI binds the network application program with the real sensors and the actuators with a button click. Since the IoTtalk application programs are implemented in Python, the IoT service is immediately provisioned without compilation when the real IoT devices are bound to the network application program.

In this paper, we extend IoTtalk with the simulation functionality by providing a simulation soft switch in the IoTtalk GUI. Before the real IoT devices are bound to the IoT applications, the developer can use SimTalk to bind the simulated sensor corresponding to a real sensor, set up specific traffic patterns to the simulated sensor to drive the IoT service, and then observe its behavior for correctness and function improvement. The paper is organized as follows. Section 2 surveys the related IoT simulation solutions. Section 3 provides an overview to the IoTtalk architecture. Section 4 proposes the SimTalk architecture based on IoTtalk. Section 5 discusses the details of SimTalk implementation for the sensors. Section 6 elaborates on the simulators for the SimTalk actuators.

\section{Related Work}

A good survey of IoT simulation is given in [12], which compared the state-of-the-art IoT simulation solutions. This survey points out that an IoT research project typically consists of two phases.

Phase 1. A proof-of-concept is realized in the virtual domain using simulation.

Phase 2. The real IoT application is implemented and experimented on a testbed.

Many simulation studies address Phase 1 in [12]. One of these studies [13] proposed agent-based adaptive parallel discrete-event simulation to enhance scalability and to permit simulation of largescale smart cities. In [14], the authors proposed YAFS (Yet Another Fog Simulator), a discrete-event fog computing simulator to model the relationships among the real IoT applications, network connections and infrastructure characteristics. In particular, the authors modeled three scenarios for dynamic allocation of new application modules, dynamic failures of network nodes and user mobility along the topology. IOTSim [15] is a simulator that enables simulation of IoT big data processing using MapReduce model in a cloud computing environment. In [16], the authors proposed an agent-oriented approach for modeling IoT networks. Specifically, by using the Omnet++ simulation platform, the ACOSO model is exploited to simulate IoT networks of various scales. Then the simulation results are used to analyze the bottlenecks at communication level. In [17], the authors also used Omnet++ to simulate the IoT applications with hardware in the loop. The resulting discrete-event simulation increases quality and significance of the modeling results, and enables the analysis of components that are not available at early stages of the development cycle.

The state-of-the-art simulation solutions described above live up to the goal of Phase 1 , and the simulation results provide very good guidelines to implement the real IoT applications in Phase 2 . However, the simulation codes of these solutions cannot be directly reused for real applications due to the "discrete event" nature of the codes (where the clock of the simulation is advanced by an event scheduler based on the timestamps of the events, which is not found in the real applications). Furthermore, since the codes of the real applications are separately developed in Phase 2, it does not guarantee that the codes in Phase 2 are consistent with the simulation codes in Phase 1. An extra verification process is required.

Unlike the above discrete-event simulation solutions, SimTalk follows the time-driven simulation approach that does not need any event scheduler used in an event-driven simulation, and can be implemented in a real IoT development environment (like IoTtalk) by using the real time advancing mechanism. With SimTalk, the development of an IoT application project seamlessly advances from Phase 1 to Phase 2 by simply changing the execution time units. There is no need to verify if the codes in both phases are consistent because they are the same in SimTalk. 
Besides simulation capability, SimTalk and IoTtalk are nicely integrated with two tools that verify the correctness of SimTalk and sensor data accuracy of IoTtalk. Such features are not found in any IoT simulators in the world. Based on the theory of bigraphs [18], correct configurations of IoT devices in SimTalk are formally verified by BigraphTalk [19], a verification framework that utilizes formal techniques to statically guarantee that there are no unwanted sensor-actuator configurations. BigraphTalk checks for invalid connections between devices, as well as type errors, e.g., passing a float to a Boolean switch. In [20,21], methods have been proposed to guarantee that the sensor data are correct. In particular, the SensorTalk approach [20] has been integrated with IoTtalk to automatically detect potential sensor failures and calibrates the aging sensors semi-automatically. When the sensors trigger the actuators, SensorTalk can detect failures within a short detection delay so that when a potential failure occurs, it is detected reasonably early without incurring too many false alarms. Details of BigraphTalk and SensorTalk are out of the scope of this paper, and can be found in $[19,20]$.

\section{The IoTtalk Architecture}

As an IoT application development platform, IoTtalk is defined in two domains [11]. In the device domain, an IoTtalk device (such as a PM2.5 sensor or a light actuator) consists of two software components:

- The Sensor and Actuator Application (SA; Figure 1a) is responsible for the implementation of the IoT device function such as the PM2.5 algorithm or the light intensity and color circuit software.

- The Device Application (DA; Figure 1b) is responsible for communications with the IoTtalk network domain. The communication technique can be wired or wireless (e.g., WiFi, LTE, NB-IoT, RoLA, and more [22]).

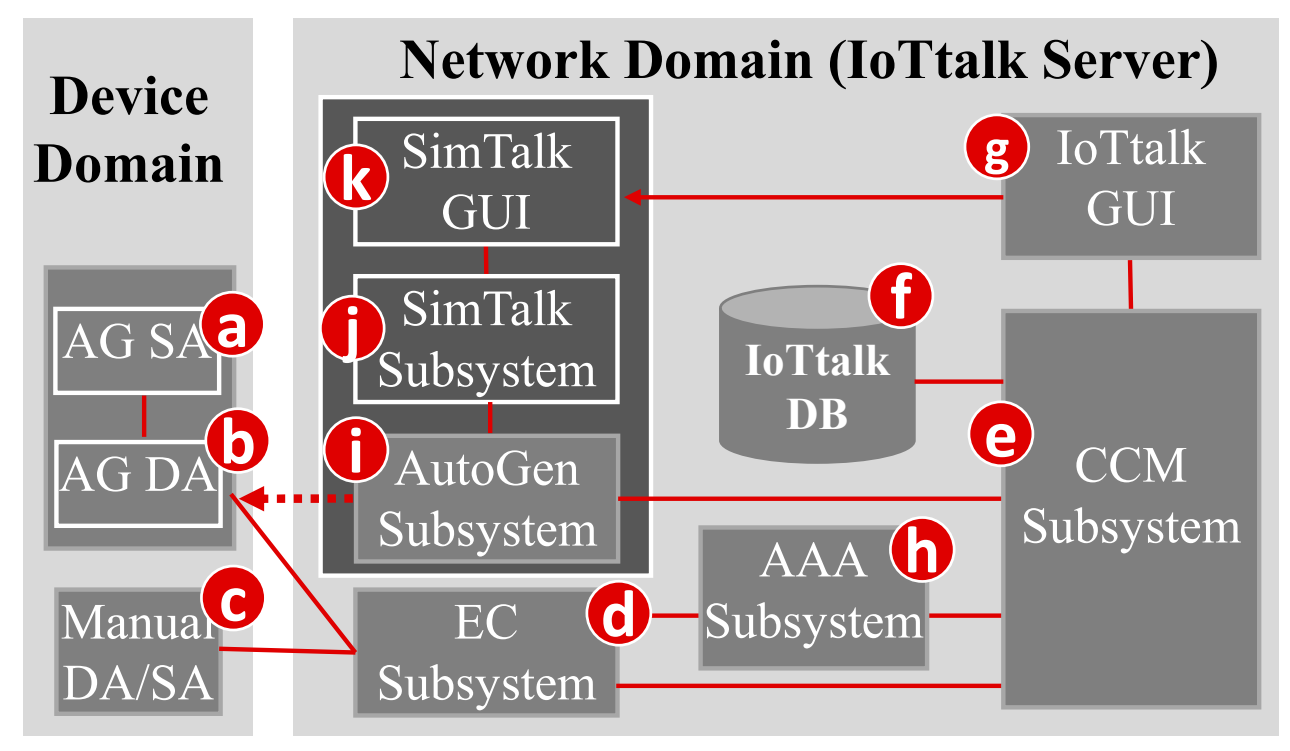

Figure 1. The IoTtalk architecture.

The DA/SA software of an IoT device can be automatically generated (AG; Figure 1a,b) or manually created (Figure 1c).

In the network domain, an IoTtalk server is responsible for provisioning the network applications that manipulate the IoTtalk devices. An IoTtalk service or project (such as smart home or smart agriculture) is a set of network applications. The server consists of several subsystems (Figure 1d-k). The Execution and Control Subsystem (EC; Figure 1d) is responsible for the control plane (the Control submodule) and the user plane (the Execution submodule) of the end-to-end path between the IoTtalk devices and the server. The Creation, Configuration and Management (CCM; Figure 1e) subsystem systematically creates and manages the network applications of the IoTtalk devices for the 
corresponding IoT services. IoTtalk defines a device model for real devices with the same properties (see Appendix A for the details). For example, a smartphone device model is mapped to various real smartphones such as iPhone, iPad, Android smartphones and more. A device model consists of several device features (DFs). For example, the acceleration sensor of a smartphone is an input DF (IDF) that sends data to the EC, and the speaker of the smartphone is an output DF (ODF) that receives instructions from the EC. For the configuration purpose, we further partition a device model into the input and the output parts. The input device model is the set of IDFs (e.g., the acceleration sensor, the gyro sensor, the camera and the soft keys of a smartphone) and the output device model is the set of ODFs (e.g., the speaker and the screen of the smartphone). The CCM is responsible for managing the device models and their DFs, and stores such information in the IoTtalk database (DB; Figure 1f). The IoTtalk GUI (Figure 1g) is a friendly web-based user interface that allows a developer to quickly establish the connections and meaningful interactions among the IoT devices. The Authentication, Authorization and Accounting Subsystem (AAA; Figure 1h) is responsible for the management of user accounts and access to the IoTtalk applications. Figure $1 \mathrm{~d}-\mathrm{h}$ are core components in a typical IoT platform, where the developer manually creates IoT devices and IoT services through the IoTtalk GUI. Two major network protocols are used in IoTtalk: Message Queueing Telemetry Transport (MQTT) are used in the links (b)-(d), (c)-(d), (d)-(e) and (e)-(g). HTTPS is used in the link (e)-(g). The DB interacts with the CCM through the ORM protocol.

Figure 2 illustrates a smart farm service where the farmer uses the weather station (Figure 2a) and the timers (Figure 2b) to control the farming actuators such as the lights (Figure 2c) and the sprayers (Figure 2d).

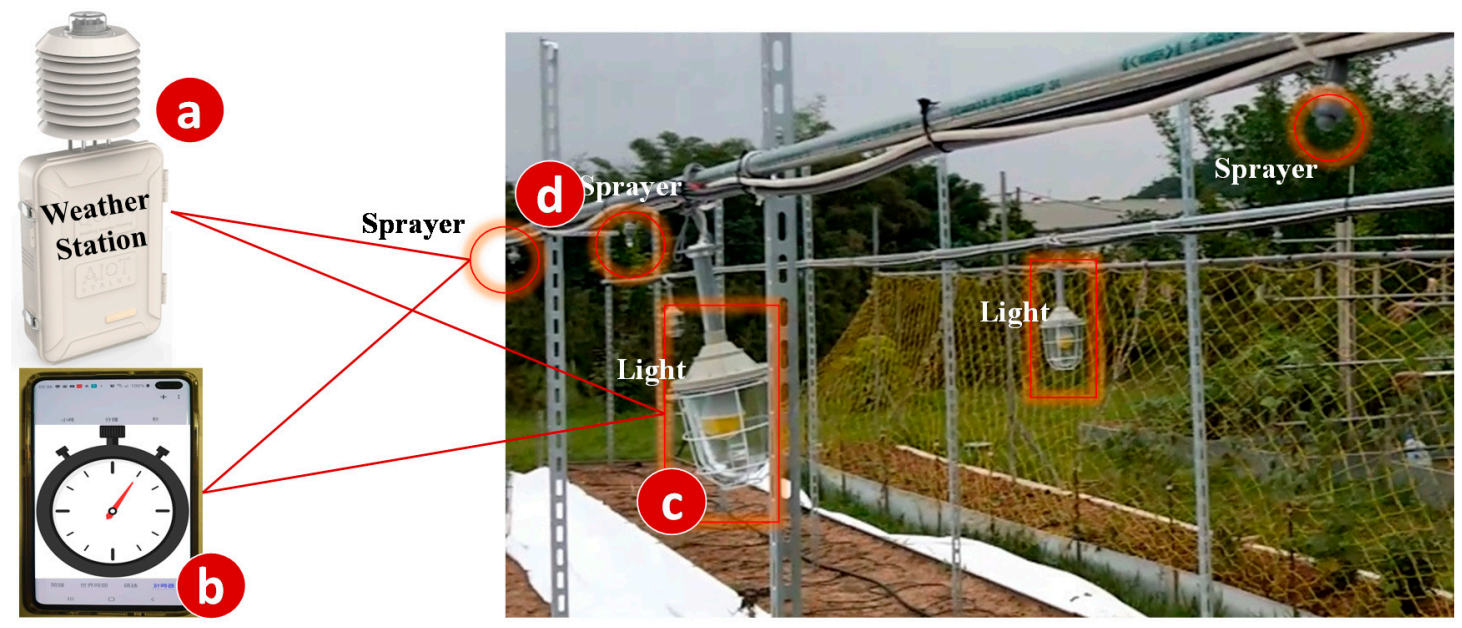

Figure 2. A simplified smart farm service.

Figure 3 shows how this simplified smart farm service is created by using the IoTtalk GUI (Figure 1g). From the model pulldown menu (Figure 3a), we select two input device models: the Sensors model (Figure 3b) implements the DA/SA for the weather station and the Timers model (Figure 3c) implements the DA/SA for multiple timers. Similarly, we select the output device model actuators (Figure $3 \mathrm{~d}$ ) that implement the DA/SA for the farming actuators. The Sensors model includes Lum-I (the IDF for the luminance sensor) and Hum-I (the IDF for the humidity sensor). The Timers model includes two IDFs for two timers. The Actuators model has Light-O (the ODF for the lights) and Spray-O (the ODF for the sprayers). To control the lights by the luminance sensor, we simply drag a line to connect Lum-I and Light-O in the GUI. In this simple smart farm service, the lights are controlled by both the luminance sensor and timer 1 through the Join 1 link. Similarly, the sprayers are controlled by both the humidity sensor and timer 2 through the Join 2 link. 


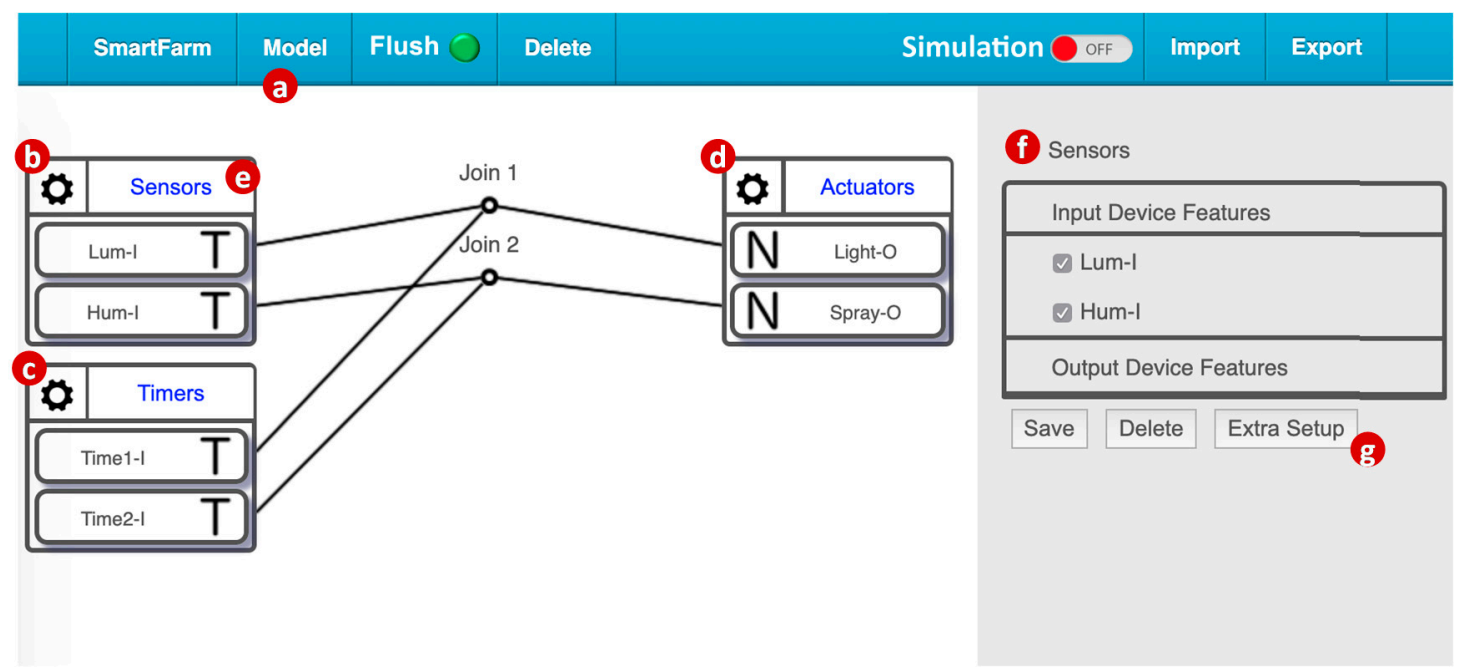

Figure 3. IoTtalk configuration for the smart farm service.

By clicking the upper-right corner of the Sensors icon (Figure 3e), the SA/DA of the Sensors model is bound to the real device, i.e., the weather station in Figure 2a, and the service is activated for execution.

\section{The SimTalk Architecture}

IoTtalk also provides an advanced feature called AutoGen, which can automatically generate devices and projects (services). An example is EduTalk [23] that is a physics and Python programming course platform. We use AutoGen to automatically create an IoTtalk project for an EduTalk course lecture to perform physics experiment through interaction with a smartphone. AutoGen is also used to interwork IoTtalk with any NB-IoT systems, where IoTtalk automatically creates a device called NB-IoTtalk for every NB-IoT service. This NB-IoTtalk device provides interaction between the NB-IoT devices (e.g., the parking sensors) with any existing IoTtalk devices to build new services [22]. Like NB-IoTtalk, AutoGen is used to interwork IoTtalk with various AI tools by packaging these AI tools as IoT devices derived from the ML_device device model [24,25]. This novel approach for integrating IoT with " $X$ " systems is provided by the AutoGen Subsystem (Figure 1i) that is considered as a platform to create "X-Talk" Subsystems (Figure 1j). In the current IoTtalk version, $X=$ Edu (education), NB-IoT, and Artificial Intelligence (AI). Every X-Talk service is associated with a web-based GUI that allows the developer to set up the parameters of the automatically generated device. For example, the developer uses AItalk GUI to select the machine-learning algorithms such as support vector machine (SVM), $\mathrm{k}$ Nearest Neighbor (kNN), Decision Tree, Random Forest, and so on. After the developer has set up the device parameters, the AutoGen Subsystem creates the device (Figure 1a,b). In this paper, we will focus on SimTalk, the simulation capability for IoTtalk, which is built on top of the AutoGen Subsystem. The SimTalk Subsystem (Figure 1j) is associated with a web-based GUI (Figure 1k) that allows the developer to set up the parameters of the automatically generated device. In Figure 1, HTTPS is used for (e)-(i) and $(\mathrm{j})-(\mathrm{k})$. The arrow link $(\mathrm{g})->(\mathrm{k})$ represents page jumps from the IoTtalk GUI to the X-Talk GUI.

The idea of SimTalk is described as follows. To further explore the AutoGen feature, for every real input device in a project, we can automatically create a counterpart simulated sensor to faithfully simulate the behavior of that input device. The detailed functional block diagram of the SimTalk and the AutoGen Subsystems in Figure 1 are illustrated in Figure 4. In this figure, the SimTalk GUI (Figure 4a) allows the developer to set up the parameters of the simulated sensors. The SimTalk Event Handler (Figure $4 \mathrm{~b}$ ) receives the instructions from the SimTalk GUI and the AutoGen Subsystem (Figure 4c), and executes the Simulator Management Procedures (Figure 4d) corresponding to these instructions. The execution results are saved in the SimTalk DB (Figure 4e). In the AutoGen Subsystem, the AutoGen Event Handler (Figure 4f) receives the instructions from the SimTalk Subsystem and the 
CCM (Figure 4g), and executes the AutoGen Management Procedures (Figure 4h) corresponding to these instructions.

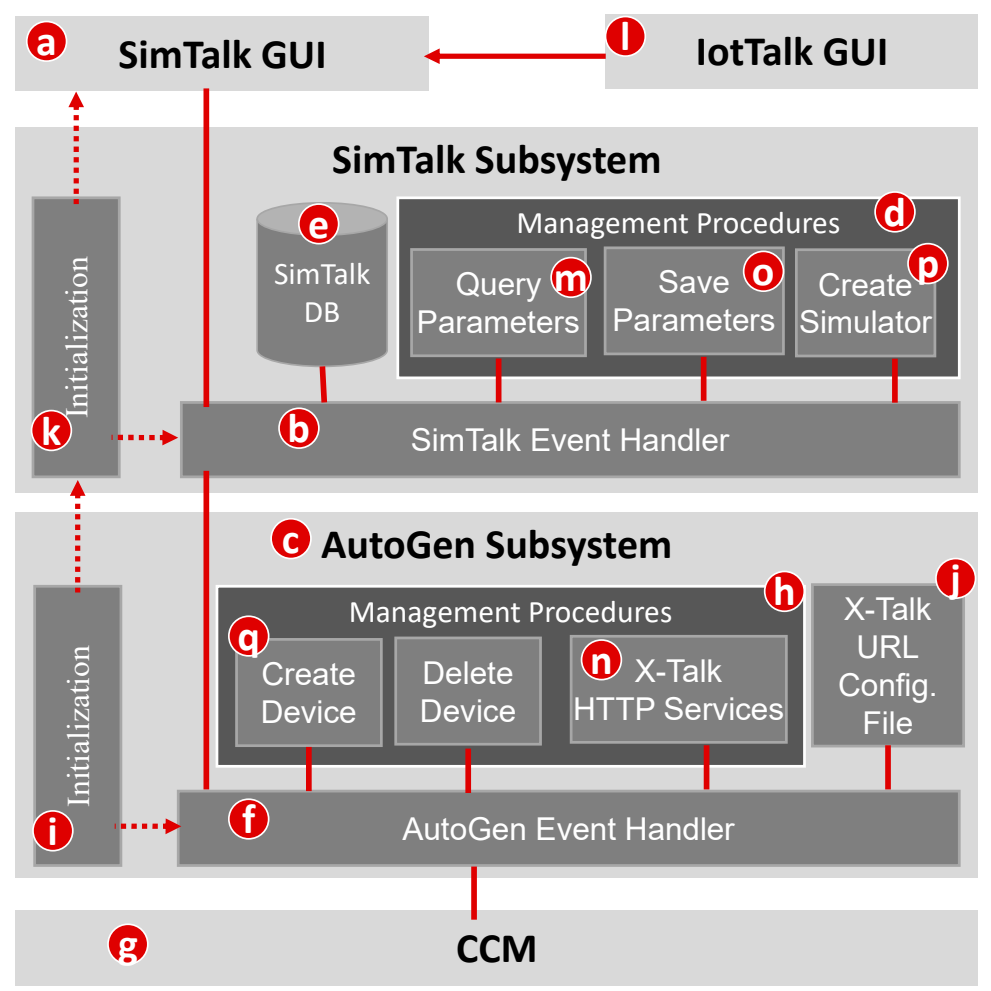

Figure 4. The SimTalk and the AutoGen Subsystems.

We note that the AutoGen Procedures are generic that apply to all X-Talks including EduTalk, NB-IoTtalk, AItalk and SimTalk.

\section{Implementing SimTalk}

When the IoTtalk server is installed, all databases including the SimTalk database (DB; Figure 4e) are initiated. When the AutoGen Subsystem is initiated, it executes the initiation program (Figure 4i) to find the host of SimTalk Subsystem in the X-Talk Configuration File (Figure 4j). This host information is used to invoke the SimTalk initialization program (Figure $4 \mathrm{k}$ ) that creates the threads for the SimTalk GUI and the SimTalk Event Handler.

We use the Sensors device model as an example to show how to set up a simulated sensor. By clicking the gear icon in the upper-left corner of Sensors (Figure 3b), the Sensors DF setup table pops up (Figure 3f). When we click the "Extra Setup" button (Figure 3g), the IoTtalk GUI (Figure 4l) jumps to the SimTalk GUI (Figure 4a). The SimTalk GUI sends a query request to the SimTalk Event Handler. The handler invokes the Query Parameters procedure (Figure $4 \mathrm{~m}$ ) to send the request to the AutoGen Event Handler. Through the X-Talk HTTP Service procedure (Figure $4 \mathrm{n}$ ), the event handler forwards the query to the CCM (Figure 4g). The CCM retrieves the parameters of IDFs (i.e., Lum-I and Hum-I) from the IoT DB (Figure 1f), and returns them to the SimTalk Subsystem through the AutoGen Subsystem. The SimTalk Event Handler invokes the Save Parameters procedure (Figure 4o) to create a record for Sensors in the SimTalk DB (Figure 4e), and saves all IDF parameters in this record.

The handler returns the Sensors record to the SimTalk GUI. This record is used to create a parameter setup window for the Sensors device model (Figure 5a), which lists Lum-I and Hum-I to be simulated. In this window, when the Lum-I button (Figure $5 b$ ) is clicked, the luminance simulated sensor setup window pops up. In this window, we set up the inter-arrival times by selecting the distribution (fixed, Exponential, Gamma, and more) through the corresponding pull down menu (Figure 5c). Similarly, 
we determine the variance and the mean of the distribution through the buttons in Figure 5d,e. If all IDFs of Sensors have the same inter-arrival times, then we can set up it by clicking the Sensors button (Figure 5a) with the similar setup procedure described above.
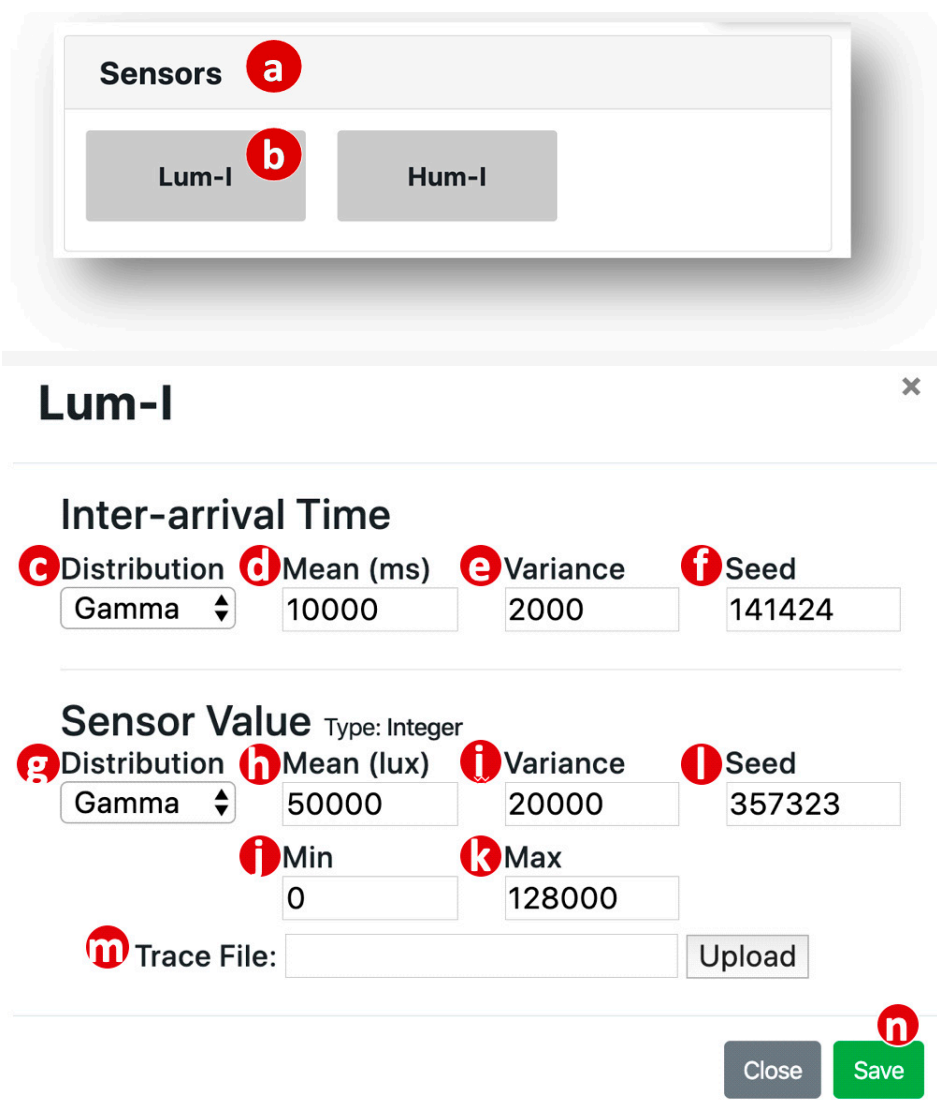

Figure 5. Web-based SimTalk graphical user interface (GUI).

We also need to set up the parameters for IDF value generation, including its distribution (Figure 5g), the mean (Figure 5h) and the variance (Figure 5i). We note that the values of some IDFs have ranges. For example, the relative luminance ranges from 0 to 128,000. For this kind of IDF, the simulated distributions must be finite or truncated to produce the values in the ranges restricted by the upper and the lower bounds. In this case, we need to fill the lower bound value (Figure 5j) and the upper bound value (Figure 5k).

To reproduce the same simulation sequence, the SimTalk GUI allows the user to specify the seeds for random number generation through the fields "Seed" (Figure 5f,l). The "Trace File" field (Figure $5 \mathrm{~m}$ ) allows the user to download a recorded trace to conduct trace-driven simulation. Note that IoTtalk records all sensor data of a real IoT application with timestamps in specified periods and saved them in a trace file. Therefore, the user can upload the trace file through the "Trace File" field. We can also perform distribution-fitting that translates the measured data in the trace file to a Gamma distribution [26]. In [3,6,10,11,22], we have manually conducted distribution fitting to make the simulation cases more valid. Implementation of automatic distribution-fitting in SimTalk will be our future work.

After the IDF setup is finished, we click the "Save" button (Figure 5n) to close the window. The SimTalk GUI passes the setup values to the SimTalk Event Handler, and the handler invokes the Save Parameters procedure to save these values into the Sensors record in the SimTalk DB.

When the user clicks the "Simulation button" in the IoTtalk GUI window (Figure 6a), a page jump occurs and the SimTalk GUI sends the "create simulators" instruction to the SimTalk Event Handler. The handler forwards the request to the CCM through the AutoGen Subsystem. The CCM queries the 
IoTtalk DB to retrieve the IDF parameter setups of all input devices in the SmartFarm project. These parameter values are returned to the AutoGen Event Handler. By invoking the X-Talk HTTP Services procedure, a response with these values is sent to the SimTalk Event Handler. The handler saves the parameter values in the SimTalk DB and invokes the Query Parameters procedure to send a response with these parameter values to the SimTalk GUI.

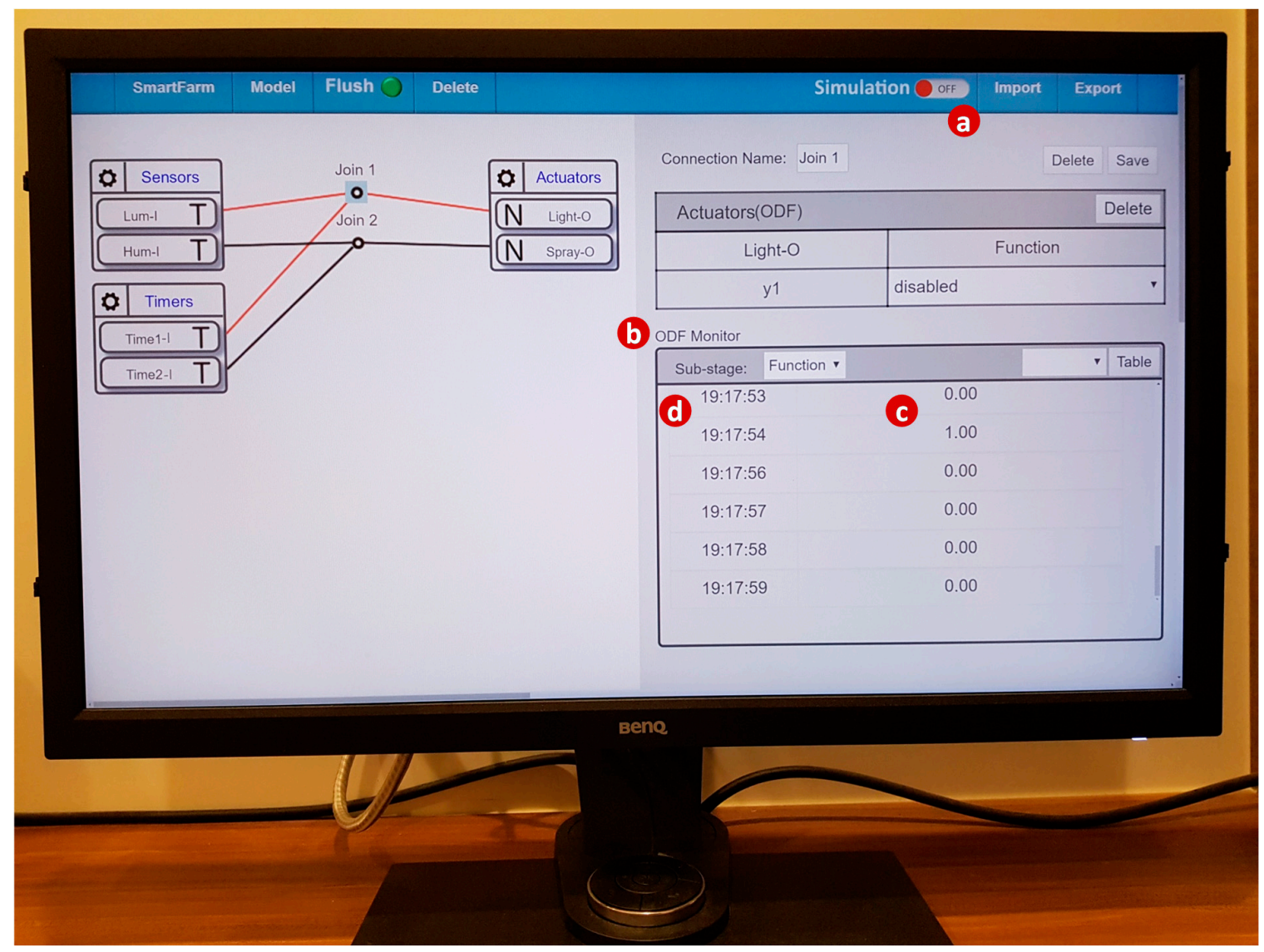

Figure 6. Web-based IoTtalk GUI (cont.).

Based on the received parameter values, the GUI pops up a window illustrated in Figure 7 to show the status of each input device model. For SmartFarm, they are Sensors and Timers. The window indicates that the Sensors device model is currently bound to a real device (Figure 7a). Therefore, the user can choose to keep the binding or unbind the real device and switch Sensors to simulation mode by clicking the simulator radio button (Figure $7 \mathrm{~b}$ ). The Timers device model is not bound to any real device, and the user did not set up the simulator parameters. Therefore, this device model is always bound to a simulated device with default parameter values (Figure 7c).

Note that the user can fill multiple values in each of the parameter text boxes (Figure $5 \mathrm{~d}, \mathrm{~h}, \mathrm{e}, \mathrm{i}$ ), and then the SimTalk simulation will sweep on these values. Each set of the values is executed for an observation period (Figure 7d). This feature is called parameter sweeping. If the parameter sweeping mode is not selected (Figure 7f), then the simulation is only executed for the first set of the values. We have designed the parameter sweeping feature for SimTalk and the implementation will be our future work. 


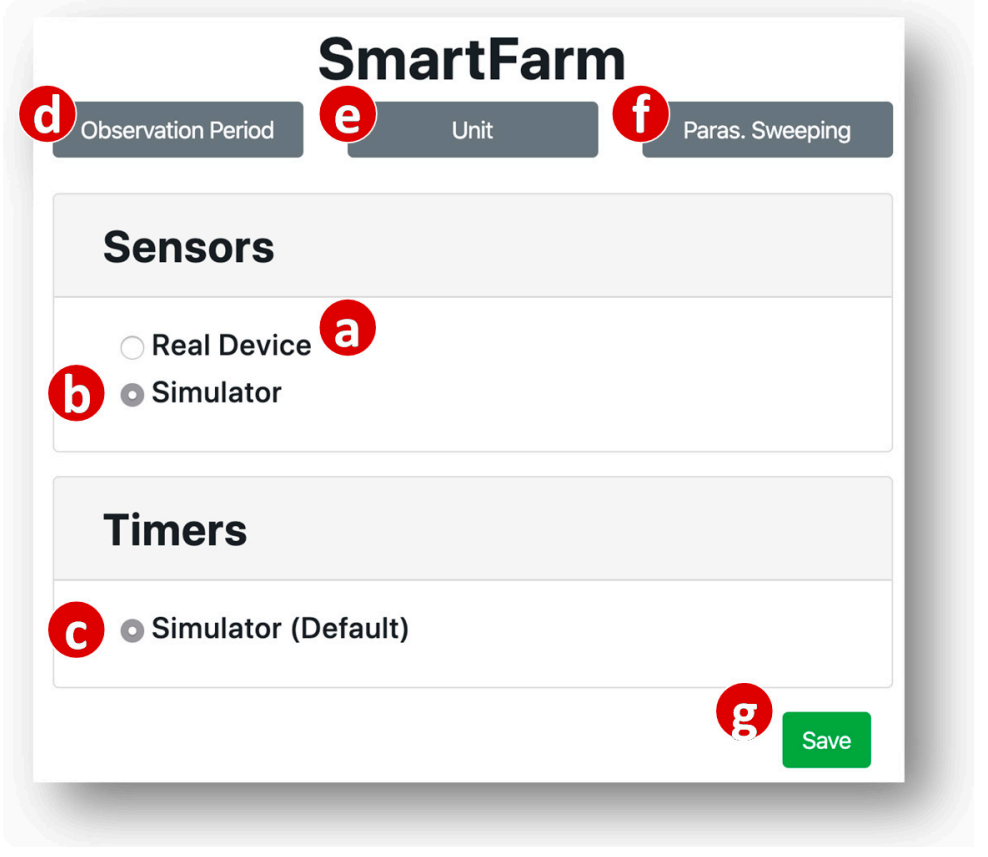

Figure 7. Web-based IoTtalk GUI (cont.).

When the user clicks the "Save" button (Figure 7g), the SimTalk GUI sends the SimTalk Event Handler a "save" request with a list of the input device model names selected as simulators. The handler saves this list in the SimTalk DB, invokes the Create Simulator procedure (Figure 4p) to generate the SA code of each simulated device (see Appendix B for the details) and requests the AutoGen Subsystem to create the simulator. The AutoGen Event Handler invokes the Create Device procedure (Figure $4 \mathrm{q}$ ) to create the devices for the simulator and sends a "bind device" request to the CCM. The CCM binds these simulated devices to the SmartFarm project and the simulation of SmartFarm is started. A successful response is sent from the CCM to the SimTalk GUI. If the simulation fails, the GUI pops up a dialog window to indicate the simulation status.

The SimTalk Subsystem reuses all IoT device codes in the IoTtalk application and inserts random number generators into the codes with the sleep() function. Furthermore, the interactions between the input devices and the output devices in SimTalk are specified in the IoTtalk GUI (Figure 3). In this way, we guarantee that the IoT device interaction in SimTalk is exactly the same as that of the real applications.

The created simulation code will be executed to conduct time-driven simulation by advancing the real clock. That is, the time advancing mechanism of SimTalk simply follows the same mechanism of IoTtalk. The progress of time in SimTalk is specified in the time interval field (Figure $5 c-f$ ), and the clock of each simulated IoT device is incremented with the time units (specified in Figure 7e) through advancing time of the real execution of IoTtalk. Therefore, SimTalk does not need any event scheduler used in the event-driven simulation. In IoTtalk, the real clock is advanced by the sleep() function in a sensor device. For example, if a temperature sensor periodically sends the measured data every $10 \mathrm{~min}$, then the temperature code simply calls sleep (600) in seconds. If we change the time unit from "minute" to "second" then SimTalk can simulate the application 60 times faster than the execution of the real application in IoTtalk. That is, the unit for the time intervals can be set up so that the progress of time is much faster than the real device execution. In SimTalk emulation, the time intervals of the simulated IoT devices must be specified exactly the same as its real device counterparts. As another example, in our work on Elevator simulation and emulation [27,28], a real elevator car takes $3.46 \mathrm{~s}$ to move up/down one floor. In the simulation, the movement of all simulated cars are delayed with the sleep() function for $0.00346 \mathrm{~s}$. In the emulation, sleep() must delay for exactly $3.46 \mathrm{~s}$ to synchronize the simulated elevator cars with the real cars. 
In a SimTalk application, if all IoT devices are simulated devices, then SimTalk conducts the simulation. If some IoT devices are real, and the remaining devices are simulated (i.e., the real devices are mixed with the simulated devices), then SimTalk conducts emulation. If all IoT devices are real, then SimTalk become IoTtalk to run real IoT applications. To switch between SimTalk and IoTtalk, one simply clicks the "Simulation" button (Figure 6a).

In the time-driven simulation, special treatments are required to simulate the sensors with very different frequencies. Suppose that we simulate $N$ sensors with the sampling periods $t_{n}, 1 \leq n \leq \mathrm{N}$, where the maximal period is $t_{M}$ and the minimum period is $t_{m}$. Then the time unit for sleep () is selected such that the central processing unit (CPU) speed of SimTalk is faster than the rate to handle the $s_{m}$ data at the frequency higher than $1 / \mathrm{E}\left[t_{m}\right]$. If the variances of the $t_{n}$ distributions are not large, then the time complexity of the execution for the time-driven simulation is about the same as the discrete-event simulation. On the other hand, if the variance of $t_{m}$ is very large, then it is possible that within two samples of $s_{M}$, there is only one $s_{m}$ sample or more than $1000 s_{m}$ samples. In the former case, SimTalk's CPU will loop in $s_{M}$ 's sleep() function for a long time. Therefore, in terms of the execution time, the event-driven approach is typically more efficient than time-driven if the events occur with high variance. Fortunately, in most IoT applications we encountered, the sensors produce data with the intervals on the same order. In the future, we will investigate the IoT applications where the events occur with very high variance, and to study how SimTalk can be modified to effectively simulate such applications.

\section{Simulating Output Devices}

In Sections 3-5, we elaborated on how to create the simulator for the input devices. Basically, these simulated devices are traffic generators that drive the IoT services for the correctness investigation. In particular, simulation results can provide useful suggestions to design physical devices for IoT field trials. For an output device model, it is important that the simulation provides two types of information:

1. The input values sent from the EC to the ODFs of the simulated output device; and

2. The results produced by the output device.

To produce the first type of information, SimTalk utilizes the "ODF monitor" in the IoTtalk GUI (Figure 6b). This monitor produces the values (Figure 6c) and their timestamps (Figure 6d) received by the ODF. The ODF monitor is a good mechanism for debugging. Besides the raw data shown in Figure $2 \mathrm{c}, \mathrm{d}$, the data can also be illustrated in the statistics charts as shown in Figure 8.

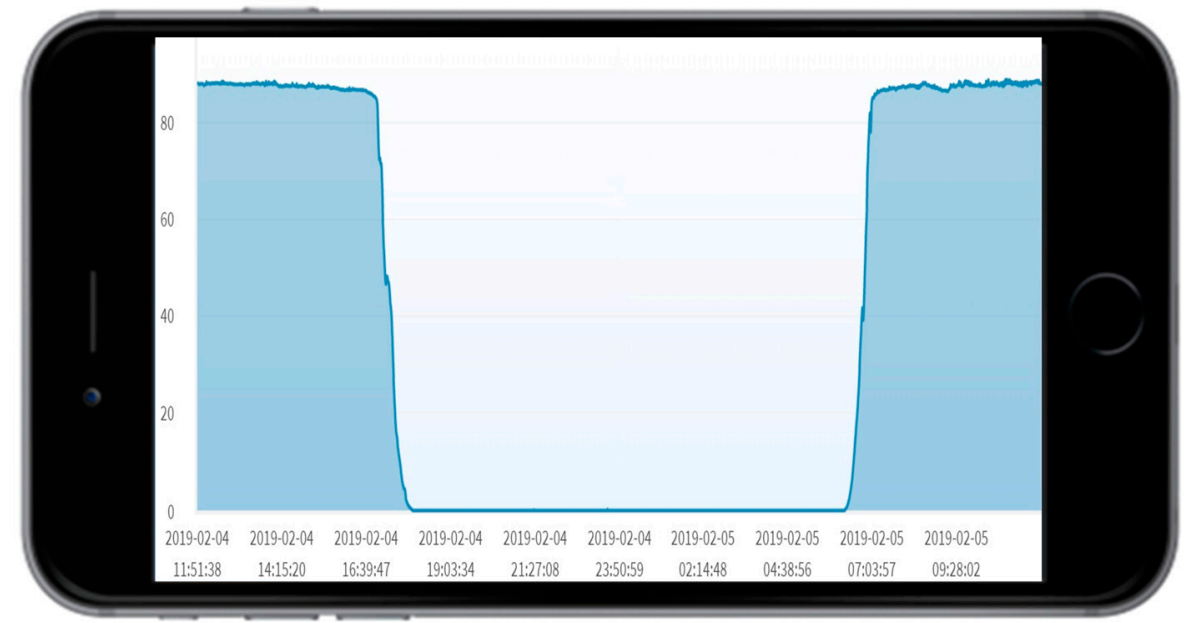

Figure 8. The time series chart of the output device feature (ODF) monitor (from Lum-I to Light-O). 
To investigate the second-type information, SimTalk also provides an animated simulator for the output device models. An example is the skeleton ceiling light that can change shape and light color during the night and reflect the shadow of different geometric shapes on the floor (Figure 9a). The shape change is achieved through compression of the skeleton stalks with various angles and sizes. Therefore, the skeleton device model has three ODFs: Angle-O, Size-O and Color-O. The skeleton simulator is an animation program that reuses SA of its physical counterpart to illustrate compression of the stalks of the skeleton with color change. The animated skeleton simulator is implemented in Java, which continuously draws the graphical skeleton patterns with specified ODF values (Figure 9b). The implementation details are given in [5].
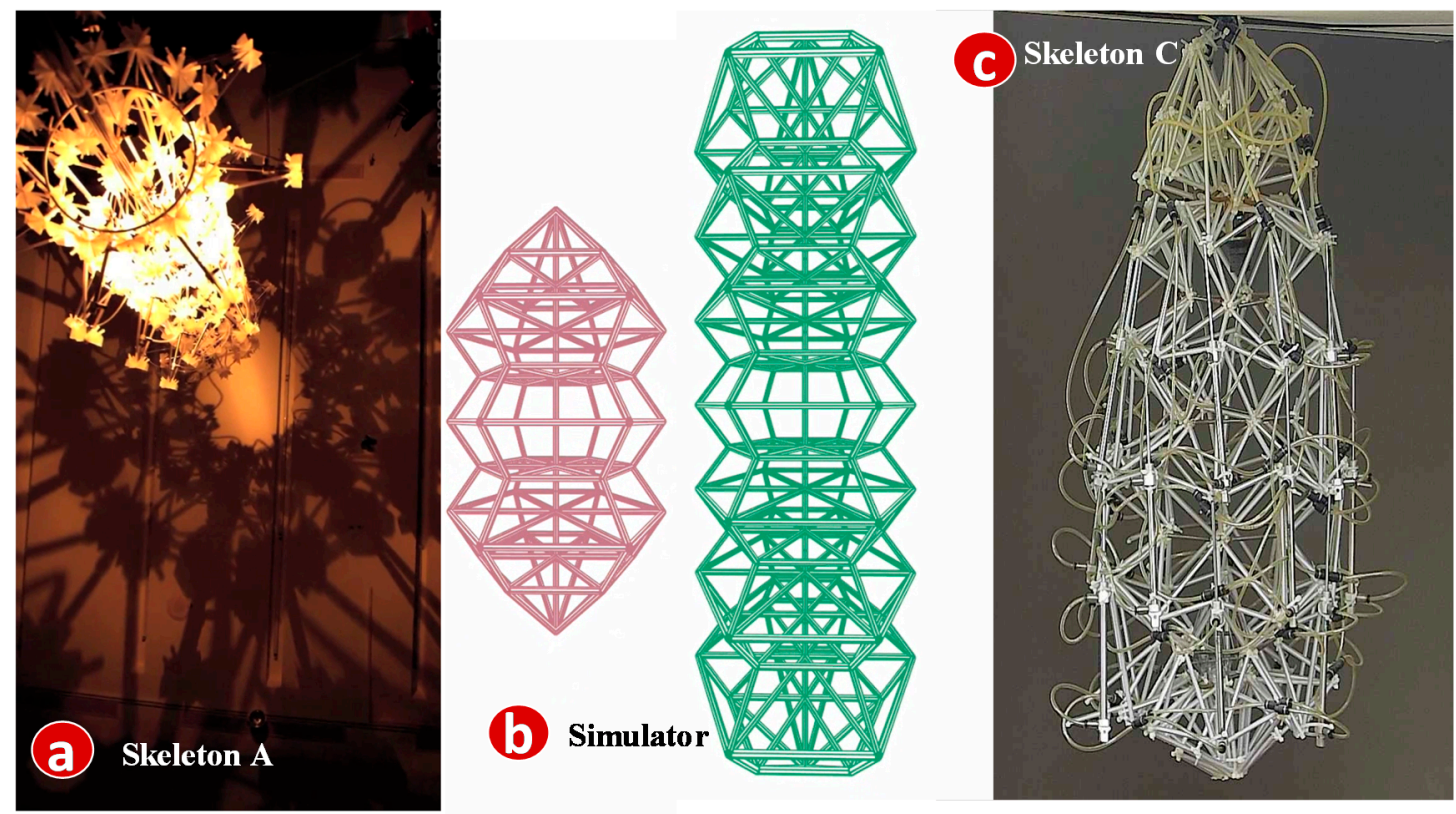

Figure 9. Skeleton: (a) geometric-shape shadow of skeleton; (b) shape change of the skeleton simulator; (c) another implementation of real skeleton device.

The skeleton device is connected to a web-based controller with three IDFs. Every IDF is a $3 \times 3$ keypad. Color-I is a 9-color palette (Figure 10c). Angle-I is a 9-number keypad (Figure 10d) that produces an angle of a decimal degree $\mathrm{n}$ when the $n$-th key is pressed, where $n=10,20, \ldots, 90$. Size-I is another 9-number keypad that produces a size $\mathrm{m}$ when the $\mathrm{m}$-th key is pressed, where $1 \leq \mathrm{m} \leq 9$. The skeleton configuration created through the IoTtalk GUI is illustrated in the right-hand side of Figure 10. A user accesses the controller (Figure 10a) through the browser of his/her smartphone. Then he/she can press the keypads to interact with the skeleton device (Figure 10b).

When the controller is switched to the simulation mode, the skeleton is driven by random sequences of the angle, the color, and the size. In Figure 10, we can add another skeleton device model (called Skeleton2) bound to a newly built Skeleton C as illustrated in Figure 9c. To ensure that Skeleton C correctly duplicates Skeleton A in Figure 9a, the simulated controller connected to Figure $9 \mathrm{~b}$ is also connected to Skeleton2. When the simulation (actually, emulation) executes the input sequences produced by the simulated controller, we can observe if Skeleton $C$ has the same behavior as the simulated Skeleton (Figure 9b). If so, Skeleton C has the same behavior as Skeleton A without any operation on Skeleton A. 


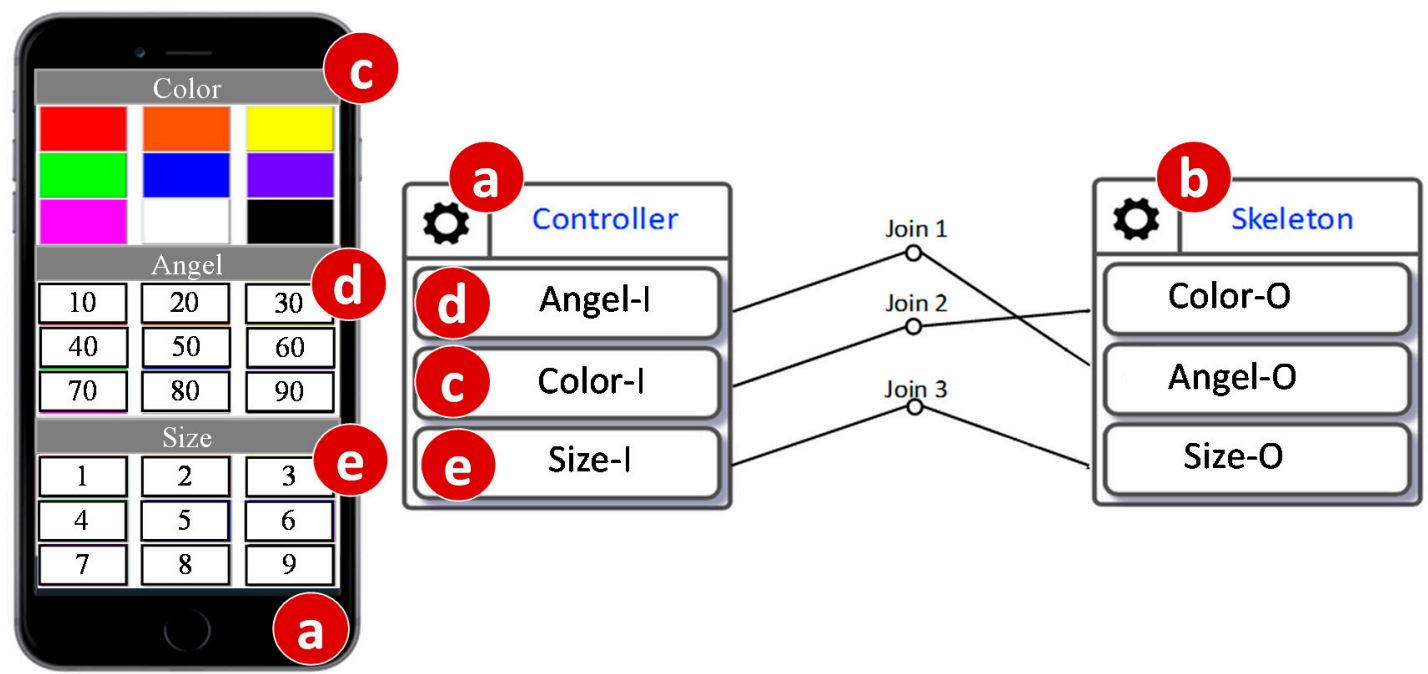

Figure 10. IoTtalk configuration for skeleton.

The results of output device simulation can provide useful suggestions to design physical devices for IoT field trials. For example, in National Chiao Tung University (NCTU), the IoTtalk platform is used to implement the water dance service in a fountain with the sprinklers and lights. Figure 11 shows that 6 simulated light bulbs are mapped to the lights under the water of the fountain. There are also 6 sprinklers and their simulated counterparts. To simplify our discussion, they are not shown. After the water-dance designer is satisfied about the control sequences of the lights and the sprinklers through the simulation (typically controlled by the music), he/she can bind the real lights and sprinklers through the IoTtalk GUI.

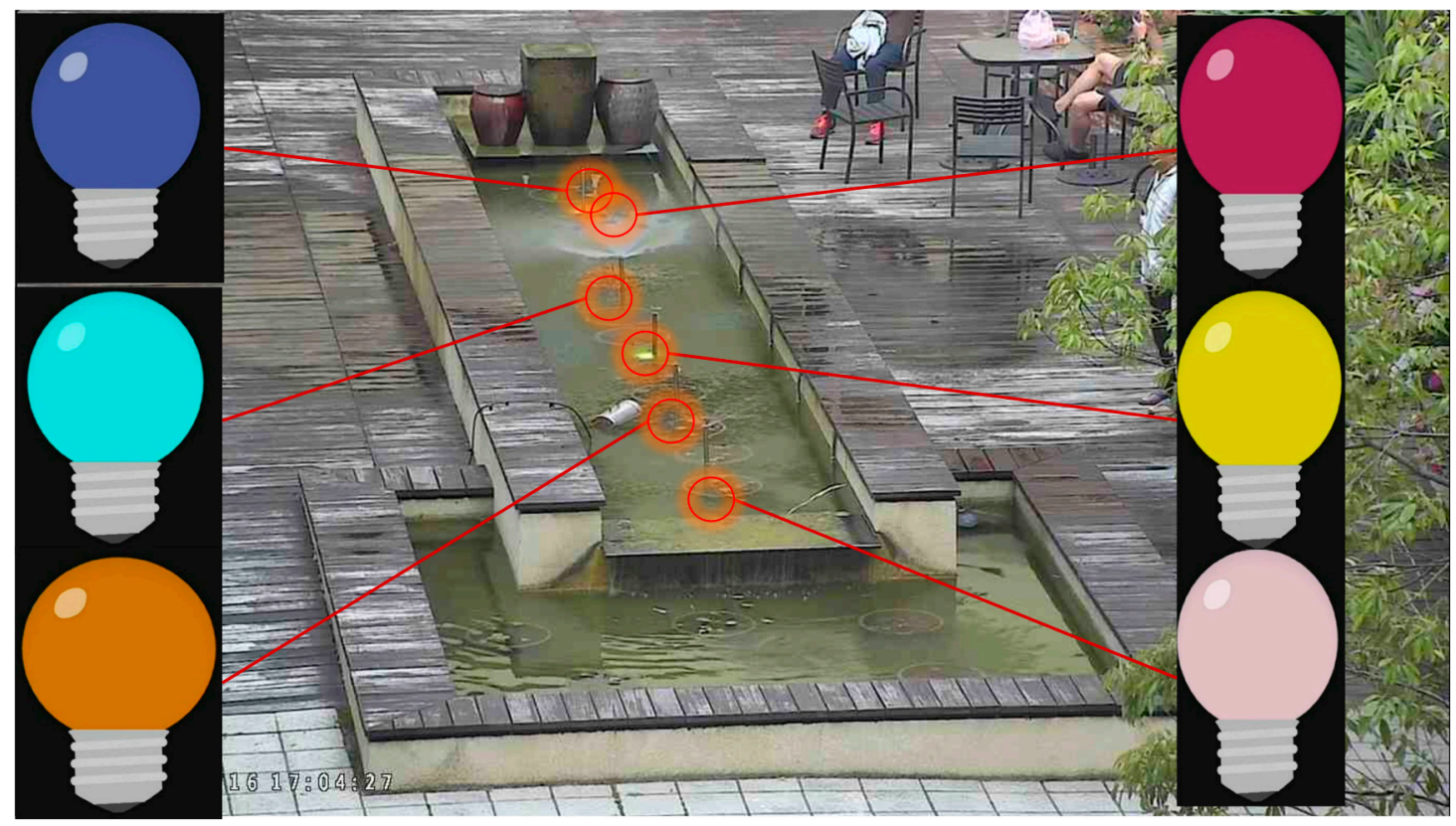

Figure 11. The real lights and the simulated lights in the water dance application.

We can also use simulated output devices to investigate the behavior of an input device. This feature is especially useful for physics experiments. Figure 12 shows how the behavior of a pendulum (Figure 12a) can be observed by its simulation counterpart (Figure 12b). 


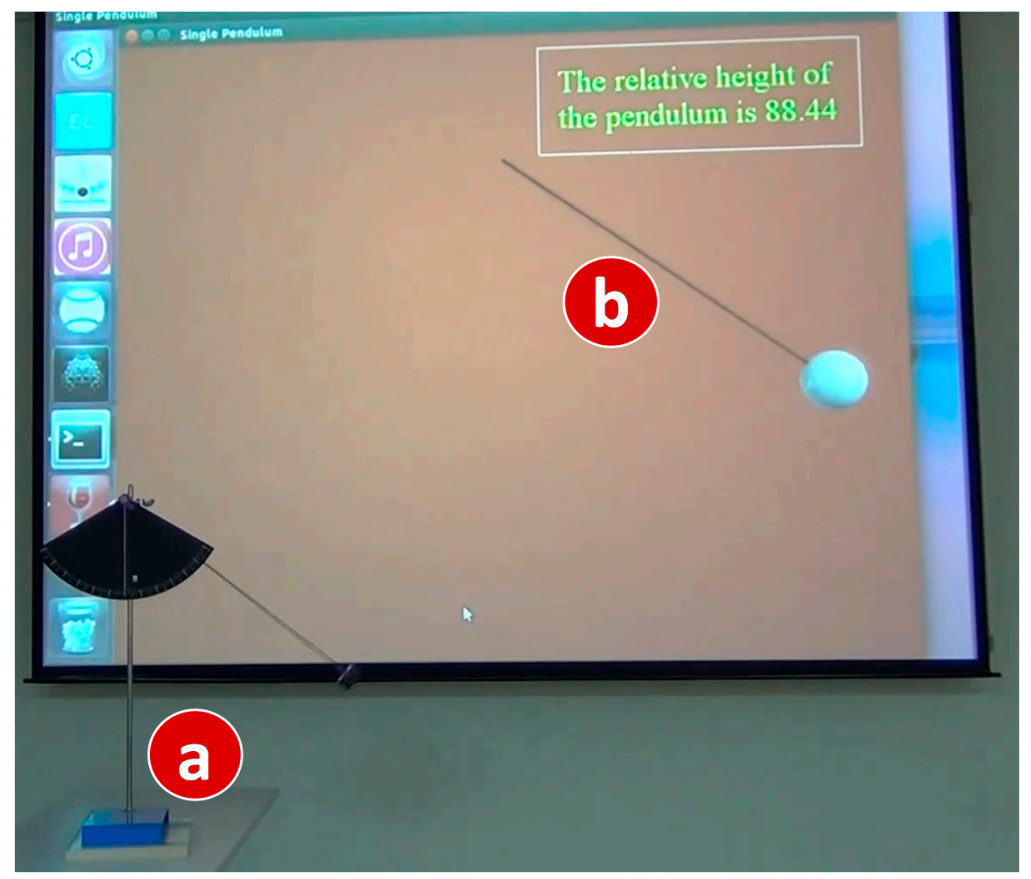

Figure 12. Simulation of pendulum.

In Figure 12, in the bob of the pendulum is installed an acceleration sensor and a WiFi/Bluetooth wireless module. The SA of the pendulum implements the IDF Acceleration-I. When the bob swings back and forth, the bob sends the acceleration values to the IoTtalk server through WiFi or Bluetooth. The IoTtalk server then links Acceleration-I to the corresponding ODF of an output simulator that is a 2-D pendulum animation written in VPython. The SA of the simulated pendulum uses the acceleration data to calculate the motion of the bob, and illustrate the bob motion through real-time animation. The animation also indicates the relative height of the pendulum. This feature is valuable because the bob position cannot be observed by the student's bare eyes from the real pendulum motion.

\section{Conclusions}

In this paper, we designed and implemented SimTalk, the simulation mechanism for an IoT application development platform called IoTtalk. We first elaborated on how to implement the simulator for an input IoT device (a sensor). We used a smart farm application to show how the simulator of sensors is used for correct implementation. Then we described how an output IoT device (an actuator) can be simulated by an animated simulator. We used applications including interactive art (skeleton art and water dance) and the pendulum physics experiment as examples to illustrate how IoT application behavior investigation can be achieved in SimTalk. A demonstration video for SimTalk is available in [29]. The source codes for developing IoT DA can be found in [30].

As the main outcome of this paper, the SimTalk simulation codes can be directly reused for real IoT applications and vice versa. Furthermore, SimTalk is nicely integrated with the tool BigraphTalk to formally verify the IoT application configuration. Such features have not been found in any IoT simulator in the world.

There are three directions for SimTalk future development:

1. Enhancing BigraphTalk to provide better simulation code verification for SimTalk;

2. Developing a distribution-fitting feature in SimTalk to replace a measured trace by a fit gamma distribution;

3. Implementing parameter sweeping to automate the execution of SimTalk with various values. 
Author Contributions: Y.-W.L.: Design and implementation of SimTalk, Y.-B.L.: Writing and Review, T.-H.Y.: Design and implementation of SimTalk. All authors have read and agreed to the published version of the manuscript.

Funding: This research was funded by the Center for Open Intelligent Connectivity from The Featured Areas Research Center Program within the framework of the Higher Education Sprout Project by the Ministry of Education in Taiwan, and Ministry of Science and Technology 108-2221-E-009-047, Ministry of Economic Affairs 107-EC-17-A-02-S5-007.

Conflicts of Interest: The authors declare no conflict of interest.

\section{Appendix A. Device Feature and Model Management}

IoTtalk provides the Device Feature and Model Management page to create and manage the device features and the device models. This page includes a "Device Feature" (DF) and a "Device Model" (DM) button (Figure A1a), the Device Feature Window (Figure A1b) and Device Model Window (Figure A1c). When the "Device Feature" mode is selected in the toggle button (Figure A1a), the Device Feature Window is activated, and the user can create a new or edit an existing device feature. On the other hand, when the "Device Model" mode is selected through the toggle button, the Device Model Window pops up to allow the user to create and edit the device model.

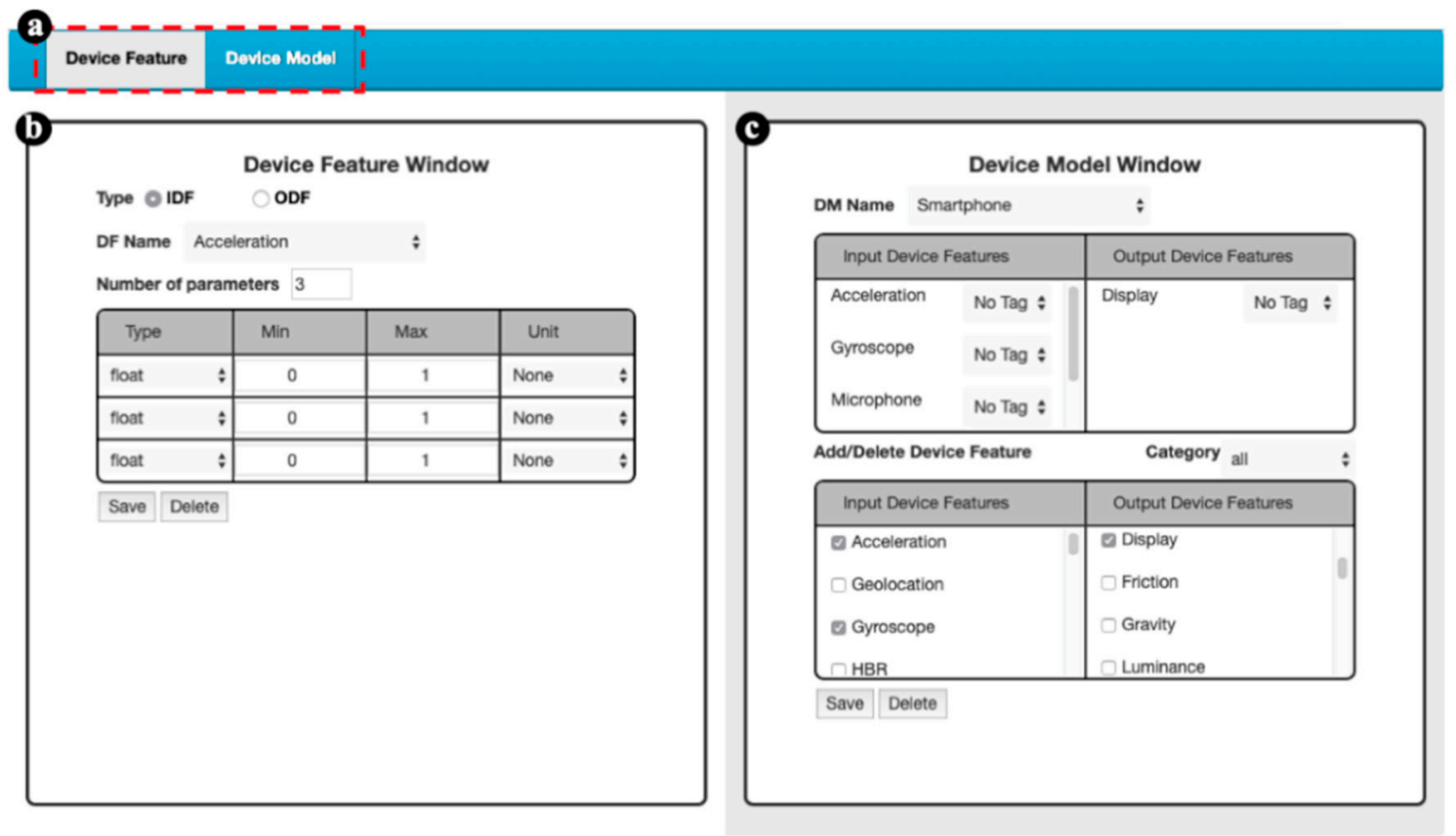

Figure A1. Device Feature and Model Management page.

\section{Appendix A.1. Device Feature Management}

To access the Device Feature Window, the user clicks the "Device Feature" button to the "Device Feature" mode.

After the "Device Feature" mode is selected, the GUI displays two radio buttons for IDF/ODF type selection (Figure A2a). The IDF type is automatically selected as the default type, and IoTtalk obtains all DFs for the selected DF type (Figure A2b). The first item of the list is "add new feature", which can be clicked to create a new DF. The details are given in the next subsection. If the user presses the ODF radio button (Figure A2a), all IDFs in the "DF Name" list (Figure A2b) are replaced by all ODFs. 


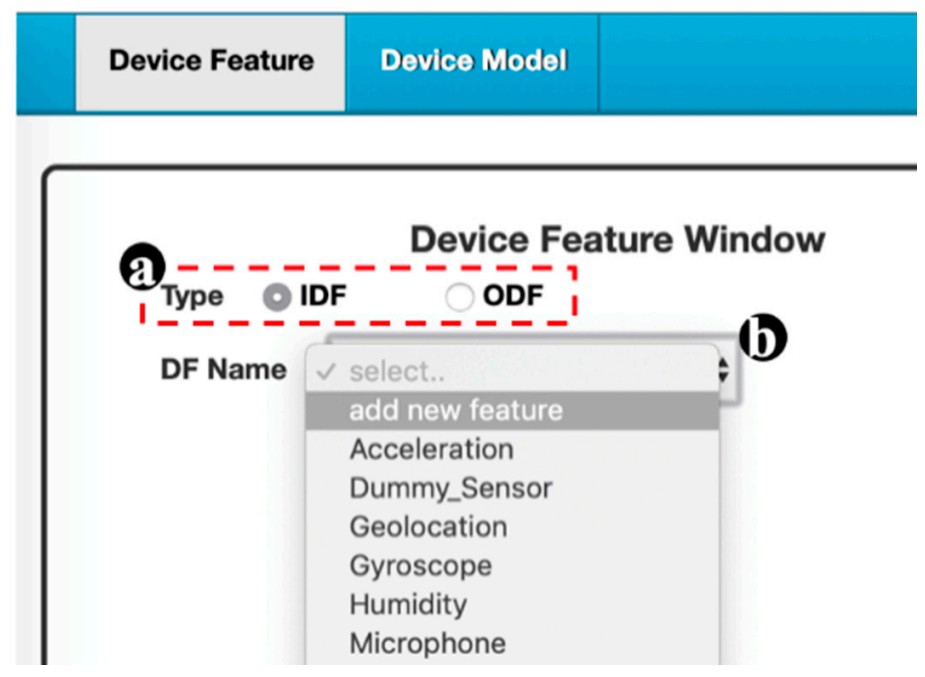

Figure A2. Device feature window.

To create a new device feature, the user selects the "add new feature" in the "DF Name" list (Figure A2b), and the GUI pops the DF Parameter module (Figure A3a) in the Device Feature Window. The rows of the DF Parameter module are created based on the number of the DF-parameters (Figure A3b). The default DF parameter number is one. For each of the DF parameters, the DF Parameter module includes the Type (i.e., the data types such as float, integer, string and so on; see Figure A3c), the Min (minimal) and Max (maximal) values (Figure A3d) and the Unit (e.g., cm, m/s ${ }^{2}$ and so on; Figure A3e). For an IDF, the Min/Max values are the boundary for the IoTtalk simulator (SimTalk) (Figure 1j). For an ODF, if the Min/Max fields are not filled, the ODF-parameters take arbitrary values without range limits. The user edits them according to the characteristics of the ODF provided in the manufacture's data sheet. For example, the luminance value of the Light-O has the range $[0,500]$. When the user clicks the "Save" button (Figure A3f), the GUI pops a dialog box for the user to input the name of the new device feature (Figure A3g). Then IoTtalk stores device feature information in the DB.

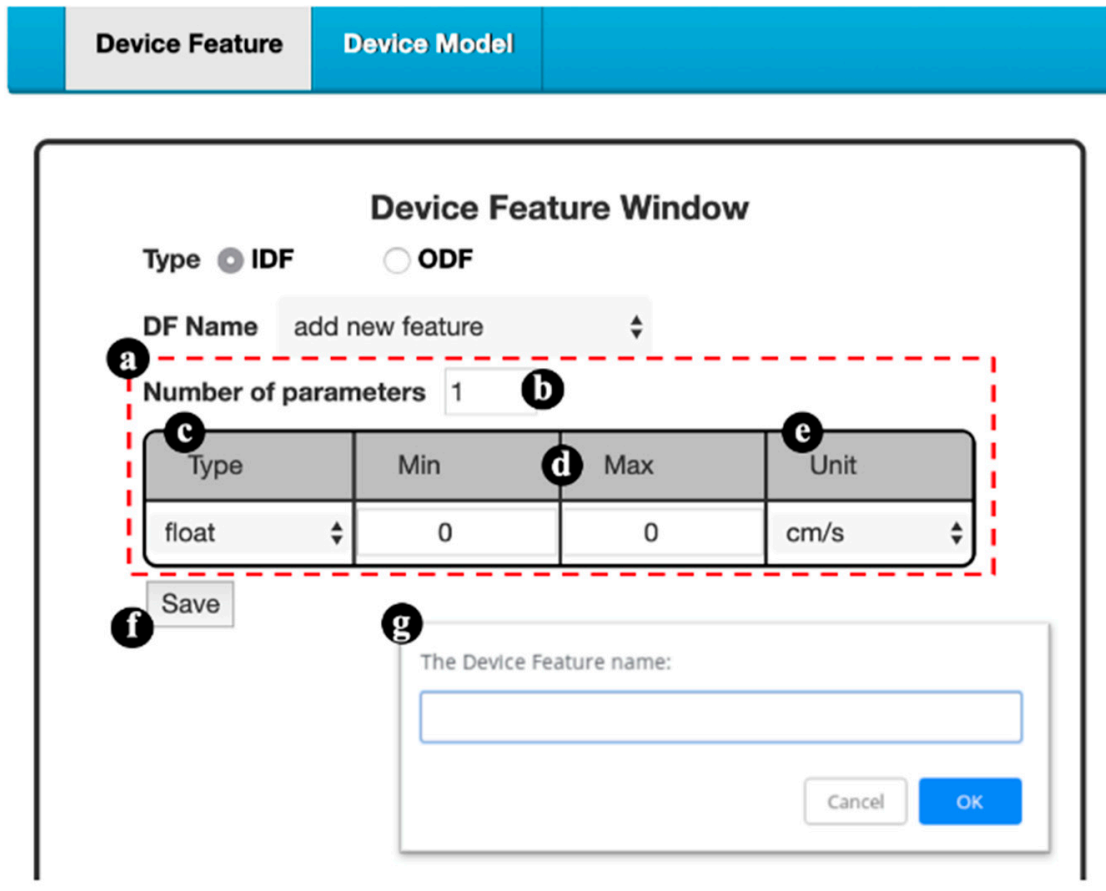

Figure A3. Device feature creation. 
From the "DF Name" list, the user selects and edits an existing DF (e.g., Acceleration; Figure A4a). If the user modifies the DF-parameter number (Figure A4b), the DF Parameter module will be redrawn based on the new number. Then the user can edit the data type, the Min/Max values and the unit of each DF-parameter. After the user has completed editing the device feature and clicks the "Save" button (Figure A4c), a dialog box pops up for the user to reconfirm the modifications (Figure A4d). The device feature name is shown in this dialog box, which allows the user to rename the modified device feature with a new one. When the user clicks the "OK" button (Figure A4e) of the dialog, IoTtalk store the information the DB. When the user clicks the "Delete" button (Figure A4f), IoTtalk removes the device feature from the DB.

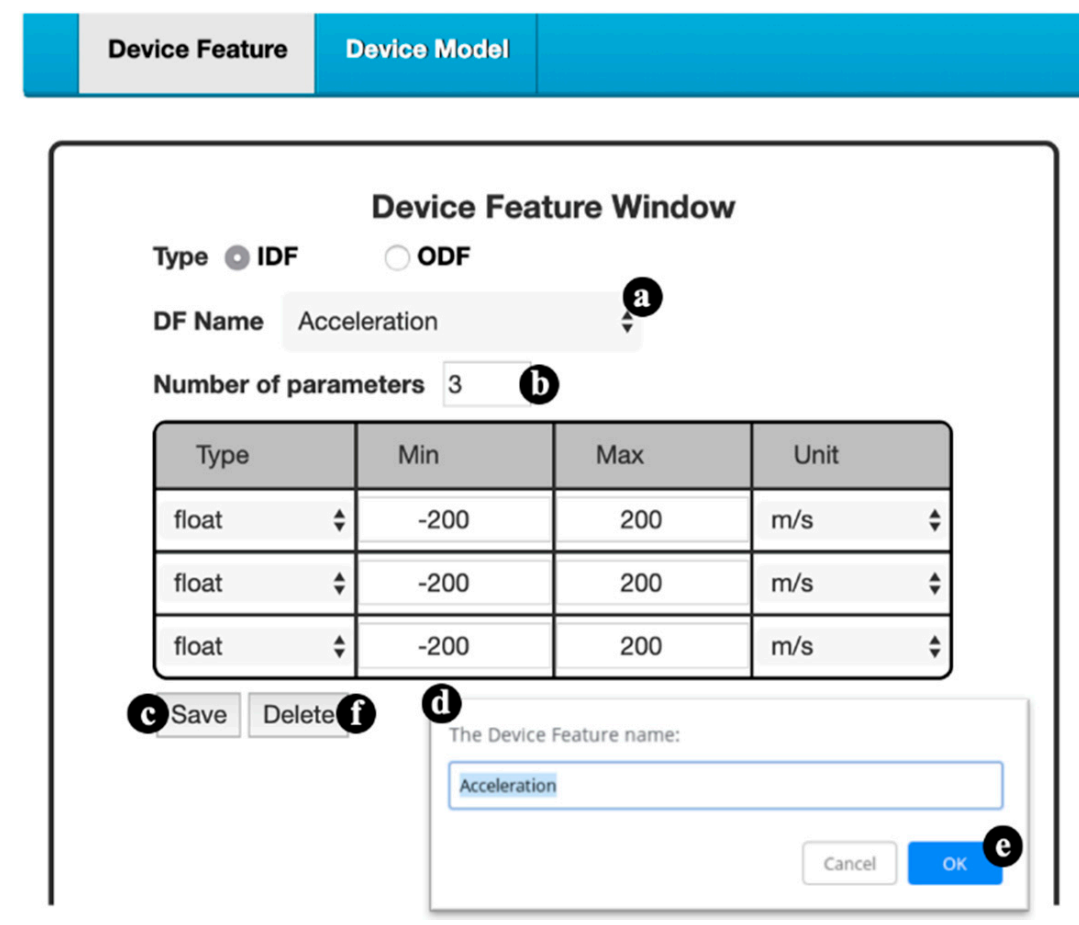

Figure A4. Device feature modification.

\section{Appendix A.2. Device Model Management}

To manage the device model, the user clicks the "Device Model" button to switch to "Device Model" mode (Figure A5a). Then the Device Model Window pops up (Figure A5b) together with Device Feature Window (Figure A5c). The Device Model Window shows the "DM Name" pull-down menu (Figure A5d) which allows the user to select a device model. The first item in the list is "add new model". This item can be clicked to create a new model. The Device Feature Window is also shown in the "Device Model" mode for the user's benefit: the user may need to know the details of a specific device feature when he/she is configuring a device model. 


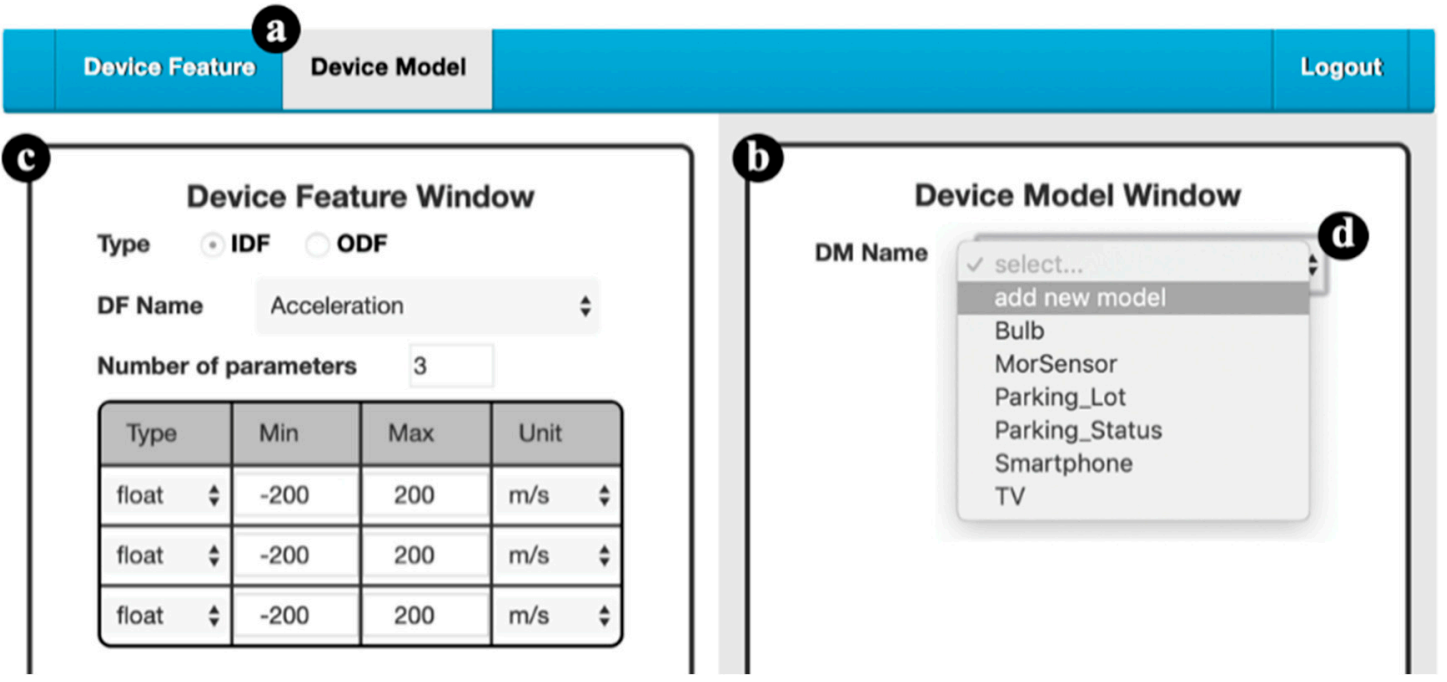

Figure A5. Device Model window.

To create a new device model, the user selects the "add new DM" in the "DM Name" pull-down menu (Figure A5d), and the GUI pops up the DF module (Figure A6a) and the "Add/Delete DF" module (Figure A6b) in the Device Model Window. The DF module lists the IDFs (Figure A6c) and the ODFs (Figure A6d) of the device model. For a new model, the DF module is empty initially. The "Add/Delete DF" module shows all IDFs (Figure A6e) and all ODFs (Figure A6f). The user can add a DF to this device model through the "Add/Delete DF" module. When the user selects a DF in the "Add/Delete DF" module, the DF is automatically displayed in the DF module (Figure A6a). After the user has selected all desired DFs for the device model and clicks the "Save" button (Figure A6g), the GUI pops a dialog box for the user to input the name of the new device model (Figure A6h) and IoTtalk create the new device model in the DB.

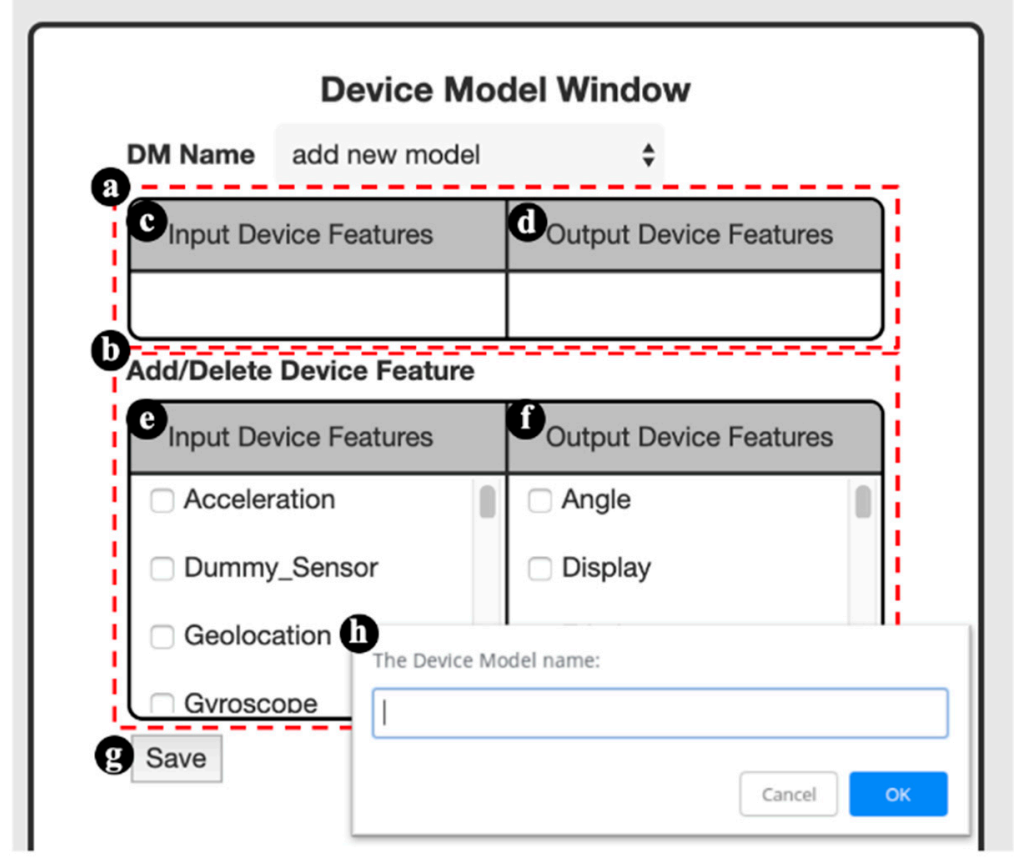

Figure A6. Device model creation.

To configure an existing device model, the user selects that device model (e.g., Smartphone; Figure A7a) in the "DM Name" pull-down menu. IoTtalk retrieves the DFs of the device model 
from the DB and the GUI pops up the DF module (Figure A7b) and the "Add/Delete DF" module (Figure A7c).

To obtain more details of a DF, the user moves the mouse pointer over the DF name in either the DF module or the "Add/Delete DF" module (e.g., Acceleration; Figure A7d). IoTtalk obtains the details of the selected device feature and the GUI shows them in the Device Feature Window (Figure A7e). In this way, the user can conveniently investigate the details of a DF selected in the Device Model Window.

If the user wants to delete a device feature (e.g., Microphone) from the device model, he/she clicks the DF item in the DF module (Figure A7f), and this device feature is shown in the "Add/Delete DF" module automatically. Then the user can unselect the device feature in the "Add/Delete DF" module (Figure A7g) to remove it from the device model.

To configure the tag parameter, each DF item in the DF module contains a pull-down menu (Figure A7h). Users can set different tags for each device feature of the device model.

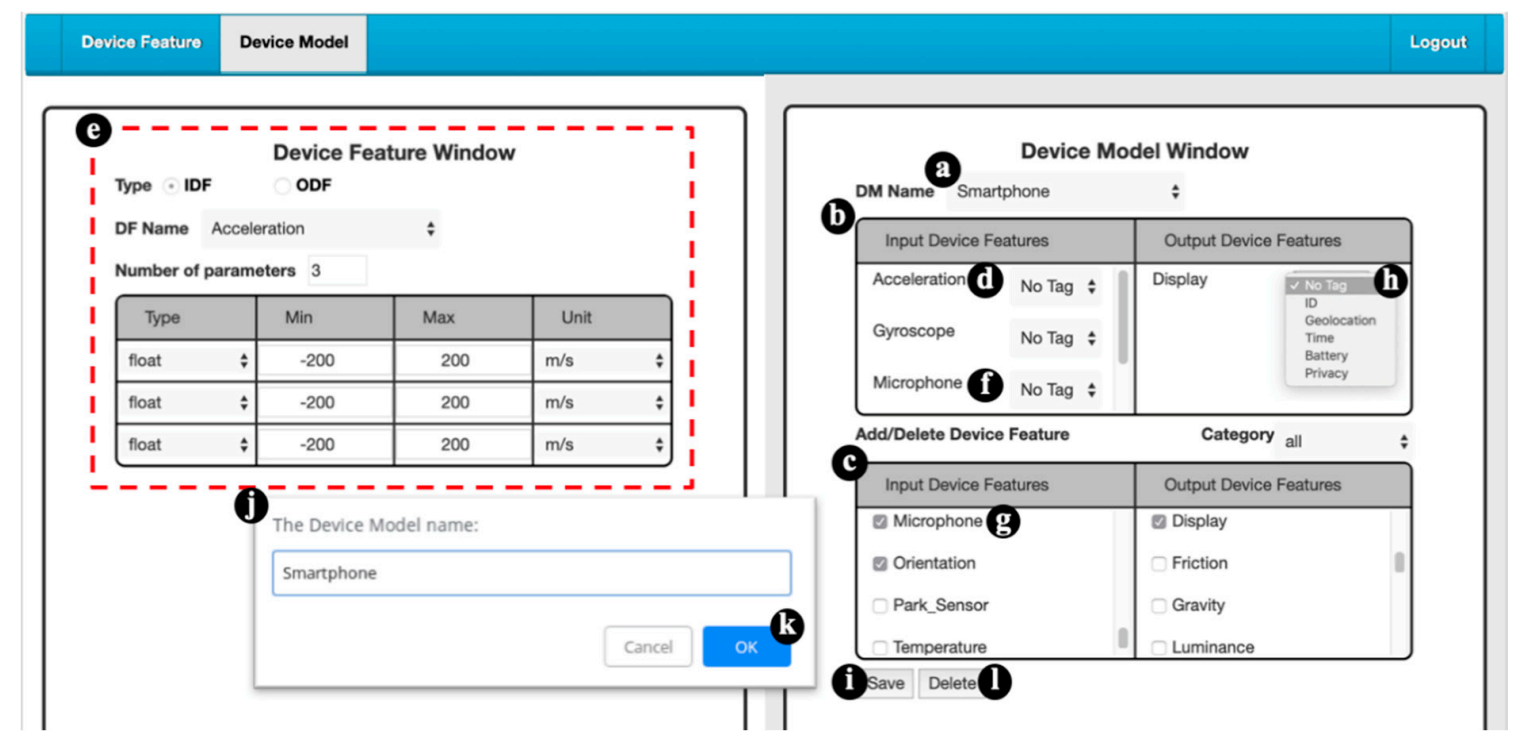

Figure A7. Device model modification.

After the user has completed configuring the device model and clicks the "Save" button (Figure A7i), a dialog pops up for the user to reconfirm the modifications (Figure A7j). The device model name is shown in this dialog, which allows the user to rename the modified device model by a new one. When the user clicks the "OK" button (Figure A7k) of the dialog, IoTtalk stores the information of the specific device model in the DB. On the other hand, when the user clicks the "Delete" button (Figure A7l), IoTtalk removes the device model from the DB.

\section{Appendix B. Creating the Simulation Codes}

Automatic creation of the simulated devices is achieved by the Create Simulator procedure of the SimTalk Subsystem (Figure 4p). We use the Sensors device as an example to show how the Create Simulator procedure generates the SA code of the simulated Sensors device. We first note that the parameters of an IDF are set up by SimTalk GUI (see Figure 5) and are stored in a record of the SimTalk DB (Figure 4e). Consider the luminance sensor (Lum-I) as an example. The format of the Lum-I parameters are listed in Figure A8, where v_paras (Lines DB.02-DB.9) describes the distribution for the sensor value generation and t_paras (Lines DB.10-DB.15) describes the distribution for the arrival time interval generation. 


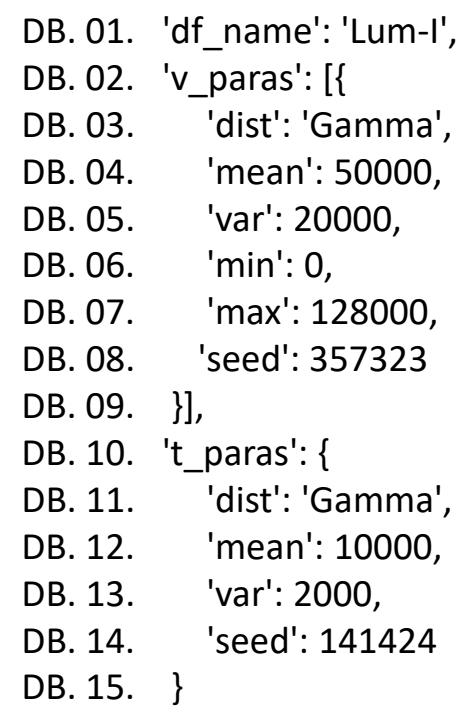

Figure A8. The Lum-I parameter record.

The Lum-I and Hum-I parameter records will be used by the Create Simulator procedure. In this procedure, Line 1 imports host (i.e., the IoTtalk server) and simulator (i.e., the simulator class in the SimTalk DB) from the config file. Line 2 imports send_event and db_session from event_handler (of the SimTalk Subsystem). The send_event function is used by the SimTalk event handler to communicate with the AG Subsystem. The class db_session supports the ORM protocol interface to query the records from the SimTalk DB.

1. from config import host, simulator

2. from event_handler import send_event, db_session

Line 3 defines the procedure create_simulator() with one argument sim_name (Sensors in our eample). Line 4 invokes the class db_session to query the IDF parameter record of sim_name from the SimTalk DB (e.g., the record described in Figure A8).

3. def create_simulator(sim_name):

4. sim_record $=d b \_s e s s i o n . q u e r y($ simulator).filter(sim_name)

Line 5 creates a list sim_paras. Lines 6-27 obtain the sensor parameters (e.g., Figure A8) from the SimTalk DB and store them in df_config. The code details are omitted. Line 28 collects the df_config records for Lum-I and Hum-I and saves them in sim_paras.

5. sim_paras $=[]$

6.-27. /*query the SimTalk DB, to obtain the df_conf records, the details are omitted*/

28. sim_paras.append(df_config)

Lines 29 and 30 load the SA text code from the file sa.py. This code is a template for the simulated device. Line 31 inserts host (i.e., the IoTtalk server), dm_name (i.e., Sensors) and the simulator parameters sim_paras (generated in Lines 5-28) into the SA code.

29. with open('sa.py') as f:

30. $\quad$ sa_code $=$ f.read ()

31. sa_code $=$ sa_code.format

(host, sim_record.dm_name, sim_paras) 
Line 32 invokes the send_event function to instruct the AutoGen Subsystem to create the simulated Sensors device.

$$
\text { 32. send_event('AG', 'create_device', sa_code) }
$$

Note that the SA text code in the sa.py file is 56 lines long and the details can be found in [21].

\section{References}

1. Shih, C.-S.; Chou, J.-J.; Reijers, N.; Kuo, T.-W. Designing CPS/IoT applications for smart buildings and cities. IET Cyber.-Phys. Syst. Theory Appl. 2016, 1, 3-12.

2. Dupont, C.; Cousin, P.; Dupont, S. IoT for Aquaculture 4.0 Smart Andeasy-to-DeployrealTimewatermonitoring with IoT. In Proceedings of the 2018 Global Internet of Things Summit (GIoTS), Bilbao, Spain, 4-7 June 2018; pp. 1-5.

3. Lin, Y.-B.; Tseng, H.-C. FishTalk: An IoT-based mini aquarium system. IEEE Access 2019, 7, 35457-35469. [CrossRef]

4. Li, X.; Lu, R.; Liang, X.; Shen, X.; Chen, J.; Lin, X. Smart community: An internet of things application. IEEE Commun. Mag. 2011, 49, 68-75. [CrossRef]

5. Lin, Y.-B.; Chen, L.-K.; Shieh, M.-Z.; Lin, Y.-W.; Yen, T.-H. CampusTalk: IoT devices and their interesting features on campus applications. IEEE Access 2018, 6, 26036-26046. [CrossRef]

6. Chen, W.-L.; Lin, Y.-B.; Lin, Y.-W.; Chen, R.; Liao, J.-K.; Ng, F.-L.; Chan, Y.-Y.; Liu, Y.-C.; Wang, C.-C.; Chiu, C.-H.; et al. AgriTalk: IoT for precision soil farming of turmeric cultivation. IEEE Internet Things J. 2019, 6, 5209-5223. [CrossRef]

7. Van, L.-D.; Lin, Y.-B.; Wu, T.-H.; Lin, Y.-W.; Peng, S.-R.; Kao, L.-H.; Chang, C.-H. PlantTalk: A smartphone-based intelligent hydroponic plant box. Sensors 2019, 19, 1763. [CrossRef] [PubMed]

8. Sulema, Y. Mulsemedia vs. Multimedia: State of the Art and Future Trends. In Proceedings of the 2016 International Conference on Systems, Signals and Image Processing (IWSSIP), Bratislava, Slovakia, 23-25 May 2016; pp. 1-5.

9. Xiao, X.; Puentes, P.; Ackermann, E.; Ishii, H. Andantino: Teaching children piano with projected animated characters. In Proceedings of the 15th International Conference on Interaction Design and Children, ser. IDC '16; ACM: New York, NY, USA, 2016; pp. 37-45.

10. Lai, W.-S.; Lin, Y.-B.; Hsiao, C.-Y.; Chen, L.-K.; Wu, C.-F.; Lin, S.-M. FrameTalk: Human and picture frame interaction through the IoT technology. Mob. Netw. Appl. J. 2019, 24, 1475-1485. [CrossRef]

11. Lin, Y.-B.; Lin, Y.-W.; Huang, C.-M.; Chih, C.-Y.; Lin, P. IoTtalk: A management platform for reconfigurable sensor devices. IEEE Internet Things J. 2017, 4, 1552-1562. [CrossRef]

12. Chernyshev, M.; Baig, Z.; Bello, O.; Zeadally, S. Internet of things (iot): Research, simulators, and testbeds. IEEE Internet Things J. 2017, 5, 1637-1647. [CrossRef]

13. D'Angelo, G.; Ferretti, S.; Ghini, V. Simulation of the Internet of Things. In Proceedings of the 2016 International Conference on High Performance Computing \& Simulation (HPCS), Innsbruck, Austria, 18-22 July 2016.

14. Lera, I.; Guerrero, C.; Juiz, C. YAFS: A simulator for IoT scenarios in fog computing. IEEE Access 2019, 7, 91745-91758. [CrossRef]

15. Zeng, X.; Garg, S.K.; Strazdins, P.; Jayaraman, P.P.; Georgakopoulos, D.; Ranjan, R. IOTSim: A simulator for analysing IoT applications. J. Syst. Archit. 2017, 72, 93-107. [CrossRef]

16. Fortino, G.; Russo, W.; Savagliom, C. Agent-Oriented Modeling and Simulation of IoT Networks. In Proceedings of the 2016 Federated Conference on Computer Science and Information Systems (FedCSIS), Gdansk, Poland, 11-14 September 2016.

17. Wehner, P.; Göhringer, D. Internet of things simulation using omnet++ and hardware in the loop. In Components and Services for IoT Platforms; Springer: Cham, Switzerland; Manhattan, NY, USA, 2017.

18. Milner, R. The Space and Motion of Communicating Agents; Cambridge University Press: Cambridge, UK, 2009.

19. Blair, A.; Shieh, M.-Z.; Hu, Y.-H.; Michele, S.; Lin, Y.-B. BigraphTalk: Verification design of IoT applications. IEEE Internet Things J. 2020. [CrossRef]

20. Lin, Y.-B.; Lin, Y.-W.; Lin, J.-Y.; Hung, H.-N. SensorTalk: An IoT device failure detection and calibration mechanism for smart farming. Sensors 2019, 19, 4788. [CrossRef] [PubMed] 
21. Hussain, S.; Mokhtar, M.; Howe, J.M. Sensor failure detection, identification, and accommodation using fully connected cascade neural network. IEEE Trans. Ind. Electron. 2014, 62, 1683-1692. [CrossRef]

22. Lin, Y.B.; Tseng, H.C.; Lin, Y.W.; Chen, L.J. NB-IoTtalk: A service platform for fast development of NB-IoT applications. IEEE Internet Things J. 2019, 6, 928-939.

23. Lin, Y.-B. EduTalk Demonstration. Available online: https://youtu.be/UCk9P3cJLkk (accessed on 29 April 2020).

24. Lin, Y.-W.; Lin, Y.-B.; Liu, C.-Y. AItalk: A tutorial to implement AI as IoT devices. IET Netw. 2019, 8, 195-202. [CrossRef]

25. Lin, Y.-W.; Lin, Y.-B.; Liu, C.-Y.; Lin, J.-Y.; Shih, Y.-L. Implementing AI as cyber IoT devices: The house valuation example. IEEE Trans. Ind. Inform. 2020, 16, 2612-2620. [CrossRef]

26. Law, A.M. How the ExpertFit Distribution-Fitting Software Can Make Your Simulation Models More Valid. In Proceedings of the 2011 Winter Simulation Conference (WSC), Phoenix, AZ, USA, 11-14 December 2011.

27. Van, L.-D.; Lin, Y.-B.; Wu, T.-H.; Lin, Y.-C. Green elevator scheduling based on IoT communications. IEEE Access 2020, 8, 38404-38415. [CrossRef]

28. Van, L.-D.; Lin, Y.-B.; Wu, T.-H.; Lin, Y.-C. An intelligent elevator development and management system. IEEE Syst. J. 2019. [CrossRef]

29. Lin, Y.-B. Simulation Demonstration. Available online: https://youtu.be/EHVdq68QteU (accessed on 29 April 2020).

30. IoTtalk. IoTtalk Learning Kit. 2020. Available online: http://iny.csie.nctu.edu.tw/\#IoTtalk (accessed on 29 April 2020).

(C) 2020 by the authors. Licensee MDPI, Basel, Switzerland. This article is an open access article distributed under the terms and conditions of the Creative Commons Attribution (CC BY) license (http://creativecommons.org/licenses/by/4.0/). 